\title{
A multi-domain boundary element analysis technique based on a row elimination-back- substitution method for solving large-scale engineering problems
}

\author{
X.-W. Gao \& J.-X. Hu \\ State Key Laboratory of Structural Analysis for Industrial Equipment, \\ Dalian University of Technology, P.R. China
}

\begin{abstract}
This paper presents a novel analysis technique using the multi-domain boundary element method (MDBEM) to solve large-scale engineering problems. Firstly, boundary integral equations for solving general heat conduction and mechanics problems are presented, which are established for a single medium and are formulated in terms of physical quantities at internal, boundary and interface points. Then a sparse system of equations formulated in terms of only interface nodal quantities is assembled based on the three-step variable condensing technique. Finally, a robust linear equation solution method is presented for solving the sparse system based on a row elimination-back-substitution method (REBSM). Combining REBSM and MDBEM makes the boundary element method more efficient for solving large practical engineering problems. A numerical example is given to demonstrate the efficiency of the proposed method.
\end{abstract}

Keywords: multi-domain boundary element method, Gaussian elimination method, row elimination-back-substitution method, sparse system of equations.

\section{Introduction}

The boundary element method (BEM) is another extensively used numerical tool in solving engineering problems after the development of the finite element method (FEM). Apart from usual advantages mentioned in references (e.g., [1]), a few important advantages of BEM over FEM can be figured out as: 1) only 
boundary of the problem needs to be discretized into elements and therefore less labor is required for preparing input data and is easy for modeling complicated problems; 2) it is efficient in solving thin-walled problems after the nearly singular integrals are accurately evaluated [2, 3]; and 3) the gradient of the basic physical quantity has the same accuracy as the physical quantity itself, since its computational formulation can be analytically derived from the basic integral equations. However, BEM has an inherent disadvantage that the formed coefficient matrices are fully-populated and non-symmetric, which limit the scale and speed of solving engineering problems.

In order to solve large-scale problems, researchers developed the multidomain boundary element method (MDBEM) [1, 4, 5]. In MDBEM, the computational domain of interesting is divided into a number of sub-domains; the BEM algebraic equations are established for each sub-domain; and the global system of equations is formed by assembling results of all sub-domains in terms of the equilibrium and consistence conditions over common interface nodes. The coefficient matrix of the global system of equations based on MDBEM is sparse, and therefore the well-developed solvers for sparse systems can be employed to solve it. The use of MDBEM not only can improve the efficiency both in problem scale and computational speed, but also can solve fracture problems by dividing sub-domains along crack surfaces [6] and multi-media problems by dividing sub-domains along interfaces [7].

In MDBEM, the assembling skill of the system of equations directly affects the computational efficiency. So far, a number of assembling techniques have been proposed $[1,4,5]$. The simple one is to put all unknowns at outer boundary nodes, and displacements and tractions at interface nodes as the unknowns of the system [1]. Such assembling technique is easy for coding, but it makes the size of the system of equations huge, limiting the capability of solving large problems. The efficient assembling technique is the variable condensing methods $[4,5]$, in which some variables are eliminated first and only a part of variables are served as the final system unknowns. Among the variable condensing methods, the three-step variable condensing technique [5] is very efficient, in which unknowns at internal and outer boundary nodes as well as tractions (or fluxes) at interfaces are eliminated in turn and only displacements (or potentials) at interface nodes are assembled as unknowns of the system. This technique can result in a smallest system of equations and the formed coefficient matrix has a higher sparsity, suitable for solving large-scale problems.

Although the system coefficient matrix of MDBEM is sparse, it is not symmetric. Therefore, the existing powerful equation solvers developed for FEM can not be borrowed to solve the MDBEM systems. New powerful solvers for sparse non-symmetric systems need to be developed. Usually, there are two types of numerical solution methods for linear systems of equations: direct and iterative methods. In direct methods, such as Gaussian elimination, Gauss-Jordan elimination, and LU-factorization methods, the answer can be obtained in a predictable number of operations [8]. In iterative methods, such as the Jacobi method, Conjugate gradients, and GMRES, many steps are necessary in attempting to converge to the desired answer [9]. To keep computational 
efficiency, the existing direct methods need to store whole coefficient matrix in core and therefore they are not suitable for solving large problems. As iterative methods are operated based on matrix-vector products, large systems can be solved. However, in iterative methods, since the approximation solutions are modified at each iterative step to approach the true answer, a convergent solution is not guaranteed for all systems of equations.

To overcome the deficiency of the existing direct methods in the requirement of large storage, a novel direct method is presented in the paper based on a row elimination-back-substitution method (REBSM). In this method, both elimination and back-substitution procedures are completed in the same row under consideration, and therefore, no later back-substitution procedure is required. Compared to the existing direct methods, such as the Gaussian elimination, the presented REBSM requires less data storage, so it can be used to solve larger system of equations. Also, since REBSM can be applied to systems of non-symmetric matrices, it is adopted in this paper to solve the MDBEM system of equations.

\section{Basic boundary integral equations in heat conduction and solid mechanics}

In this paper, the heat conduction and solid mechanics problems are served as the research backgrounds. However, the results can be extended to other problems.

\subsection{General boundary-domain integral equations for heat conduction}

The control equation for general heat conduction problems can be expressed as

$$
\frac{\partial}{\partial x_{i}}\left(k_{\mathrm{ij}} \frac{\partial u}{\partial x_{j}}\right)+Q=0
$$

where $u$ is the temperature, $k_{\mathrm{ij}}$ and $Q$ are heat conductivities and source, respectively. $k_{\mathrm{ij}}$ may be the function of coordinates $x_{i}$ and temperature $u$ in non-homogeneous and non-linear problems. The general boundary-domain integral equation for eqn (1) can be derived from the source isolation method [10] as

$$
k u+\int_{\Gamma} q^{*} u d \Gamma=-\int_{\Gamma} G q d \Gamma+\int_{\Omega} V u d \Omega+\int_{\Omega} G Q d \Omega
$$

where $\Gamma$ represents the boundary of the computational domain $\Omega, G$ is the Green's function, and $q$ is the heat flux.

$$
G= \begin{cases}\frac{1}{2 \pi} \ln \left(\frac{1}{r}\right) & \text { for } 2 D(\beta=2) \\ \frac{1}{4 \pi r} & \text { for } 3 D(\beta=3)\end{cases}
$$


156 Boundary Elements and Other Mesh Reduction Methods XXXIII

$$
\begin{gathered}
q=-k_{\mathrm{ij}} \frac{\partial u}{\partial x_{j}} n_{\mathrm{i}} \\
q^{*}=\frac{\partial G}{\partial x_{i}} k_{\mathrm{ij}} n_{\mathrm{j}} \\
k=\sum_{i=1}^{\beta} k_{i i} / \beta \\
V=\frac{\partial}{\partial x_{i}}\left(k_{\mathrm{ij}} \frac{\partial G}{\partial x_{j}}\right)=\frac{\partial k_{\mathrm{ij}}}{\partial x_{i}} \frac{\partial G}{\partial x_{j}}+k_{\mathrm{ij}} \frac{\partial^{2} G}{\partial x_{i} \partial x_{j}}
\end{gathered}
$$

From eqn (4b) it can be seen that the coefficient $k$ in eqn (2) is the average value of the diagonal terms of $k_{\mathrm{ij}}$. For isotropic problems with constant material parameters, $k$ is reduced to the usual conductivity, while $V=0$.

\subsection{General boundary-domain integral equations for mechanics}

The equilibrium equation for solid mechanics can be expressed as

$$
\sigma_{i j, j}+b_{i}=0
$$

where $b_{i}$ is the body force. The relationship between the stress $\sigma_{\mathrm{ij}}$, strain $\varepsilon_{\mathrm{ij}}$ and displacement $u_{i}$ is [11]

$$
\sigma_{i j}=D_{i j k l} \varepsilon_{k l}=D_{i j k l} u_{k, l}
$$

in which $D_{i j k l}$ is the stress-strain constitutive tensor which is symmetric about subscripts, i.e., $D_{i j k l}=D_{j i l k}=D_{k l i j}$. For non-homogeneous materials or nonlinear problems, $D_{i j k l}$ may be the functions of coordinates or stresses. From the source isolation method [10], the boundary-domain integral equations for eqn (6) can be derived as:

$$
\mu_{i j} u_{j}+\int_{\Gamma} T_{i j} u_{j} d \Gamma=\int_{\Gamma} U_{i j} t_{j} d \Gamma+\int_{\Omega} V_{i j} u_{j} d \Omega+\int_{\Omega} U_{i j} b_{j} d \Omega
$$

where $U_{i j}$ is the Kelvin displacement fundamental solutions, $t_{i}$ is the traction:

$$
U_{i j}=\left\{\begin{array}{cc}
\frac{1}{8 \alpha \pi(1-v)}\left\{-(3-4 v) \ln (r) \delta_{i j}+r,{ }_{i} r,{ }_{j}\right\} & \text { for } 2 D(\alpha=1) \\
\frac{1}{8 \alpha \pi(1-v) r}\left\{(3-4 v) \delta_{i j}+r,{ }_{i} r,{ }_{j}\right\} & \text { for } 3 D(\alpha=2) \\
T_{i j}=U_{i k, l} D_{k l j s} n_{s} &
\end{array}\right.
$$




$$
\begin{gathered}
\mathrm{V}_{\mathrm{ij}}=\left(U_{i k, l} D_{k l j s}\right)_{, s}=U_{i k, l} D_{k l j s, s}+U_{i k, l s} D_{k l j s} \\
\mu_{i j}=\frac{1}{2 \beta \chi}\left[2(\chi-1) D_{i s j s}-D_{i j s s}\right]
\end{gathered}
$$

in which $n_{s}$ is the outward normal, $\chi=(\beta+2)(1-v)$ with $v$ being the Poison's ratio. From eqn (10) it can be seen that the coefficient $\mu_{i j}$ in eqn (7) is symmetric, that is $\mu_{i j}=\mu_{j i}$. For isotropic elasticity problems with constant material parameters, $\mu_{i j}=\mu \delta_{\mathrm{ij}}$ with $\mu$ being the shear modulus, and $\mathrm{V}_{\mathrm{ij}}=0$.

It is noted that, in coding, all domain integrals appearing in eqns (2) and (7) are transformed into boundary integrals using the radial integration method (RIM) [12], resulting in a cell-less BEM analysis scheme.

\section{MDBEM based on three-step variable condensation method}

The boundary-domain integral equations presented above are derived for single domain problems. However, practical engineering problems usually are composite structures consisting of different materials. To solve such problems, the multi-domain boundary element method (MDBEM) usually is employed $[1,4]$. For this purpose, the three-step variable condensing MDBEM [5] is adopted in this study. Thus, the domain of problem is divided into a number of sub-domains. For each sub-domain, nodes are classified into three types: selfnodes (not shared with other sub-domains), common interface nodes, and internal nodes. Integral equations (2) and (7) are applied to the three types of nodes, and following algebraic matrix equations can be established for each subdomain.

$$
\begin{aligned}
& A_{b s} x_{s}+H_{b c} u_{c}+H_{b i} u_{i}=y_{b}+G_{b c} t_{c} \\
& A_{i s} x_{s}+H_{i c} u_{c}+H_{i i} u_{i}=y_{i}+G_{i c} t_{c}
\end{aligned}
$$

in which subscripts $s, c$ and $i$ represent quantities corresponding to the self, common and internal nodes, respectively; $b={ }_{s}+c$ denotes self plus common nodes related to current sub-domain; $x_{s}$ is the unknown vector consisting of all unknown displacements and unknown tractions at the self nodes of the subdomain, and $y_{b}$ and $y_{i}$ are known vectors formed by multiplying specified displacements and tractions with corresponding elements of related coefficient matrices.

First step: Eliminating internal displacements $u_{i}$ from eqns (11) and (12), it follows

$$
\bar{A}_{b s} x_{s}+\bar{H}_{b c} u_{c}=\bar{y}_{b}+\bar{G}_{b c} t_{c}
$$

where 
158 Boundary Elements and Other Mesh Reduction Methods XXXIII

$$
\begin{aligned}
& \bar{A}_{b s}=A_{b s}-H_{b i}\left(H_{i i}\right)^{-1} A_{i s} \\
& \bar{H}_{b c}=H_{b c}-H_{b i}\left(H_{i i}\right)^{-1} H_{i c} \\
& \bar{G}_{b c}=G_{b c}-H_{b i}\left(H_{i i}\right)^{-1} G_{i c} \\
& \bar{y}_{b}=y_{b}-H_{b i}\left(H_{i i}\right)^{-1} y_{i}
\end{aligned}
$$

Second step: Noticing that $b=s+c$ and eliminating unknowns $x_{s}$ in eqn (13) yield

$$
\hat{H}_{c c} u_{c}=\hat{y}_{c}+\hat{G}_{c c} t_{c}
$$

where

$$
\begin{aligned}
& \hat{H}_{c c}=\bar{H}_{c c}-\bar{A}_{c s}\left(\bar{A}_{s s}\right)^{-1} \bar{H}_{s c} \\
& \hat{G}_{c c}=\bar{G}_{c c}-\bar{A}_{c s}\left(\bar{A}_{s s}\right)^{-1} \bar{G}_{s c} \\
& \hat{y}_{c}=\bar{y}_{c}-\bar{A}_{c s}\left(\bar{A}_{s s}\right)^{-1} \bar{y}_{s}
\end{aligned}
$$

Third step: Eliminating common nodal tractions and forming the system of equations.

Equation (15) holds true for every sub-domain. For assembling the global system, it is written as the following form for the $n$-th sub-domain:

$$
t_{c}^{(n)}=\left(\hat{G}_{c c}^{(n)}\right)^{-1}\left(\hat{H}_{c c}^{(n)} u_{c}^{(n)}-\hat{y}_{c}^{(n)}\right)
$$

The common nodal displacement vector $u_{c}^{(n)}$ for the $n$-th sub-domain can be expressed in terms of the globally numbered common displacement vector $X$ by using a transfer matrix $Q^{(n)}$ as

$$
u_{c}^{(n)}=Q^{(n)} X
$$

The transfer matrix $Q^{(n)}$ consists of 0 and 1 , determined by the consistent condition of displacement at the common nodes. Considering contributions of all sub-domains at common nodes, the equilibrium condition of the traction states that

$$
\sum_{n} t_{c}^{(n)}=0
$$

Substituting eqn (18) into (17), and the result into (19), the following system of equations can be obtained

$$
A X=B
$$

where

$$
\begin{gathered}
A=\sum_{n}\left(\hat{G}_{c c}^{(n)}\right)^{-1} \hat{H}_{c c}^{(n)} Q^{(n)} \\
B=\sum_{n}\left(\hat{G}_{c c}^{(n)}\right)^{-1} \hat{y}_{c}^{(n)}
\end{gathered}
$$

Solving eqn (20) for all interface nodal displacements and substituting them back to previous expressions, one can obtain all unknowns. It is noted that the matrix 
$A$ in eqn (20) is a non-symmetric sparse matrix and, therefore, an equation solver for such systems needs to be developed. The row elimination-back-substitution method( REBSM) described in the following section is an effective one for solving such type of problems.

\section{Row elimination-back-substitution method (REBSM) for solving non-symmetric sparse linear systems of equations}

The system of equation (20) can be expressed as the following form:

$$
\sum_{j=1}^{n} a_{i j} x_{j}=b_{i}
$$

where $n$ is the order of the equation set.

The key idea of REBSM is to find solutions of the system by completing both elimination and back-substitution procedures within each row. The main advantage of this treatment over the Gaussian elimination method is the less storage requirement of intermediate data. It is assumed that, after the treatment of the first $k-1$ rows, the following expressions have been obtained

$$
x_{i}=b_{i}^{(k-1)}-\sum_{j=k}^{n} a_{i j}^{(k-1)} x_{j} \quad(i=1,2, \ldots, k-1)
$$

For the $k$-th equation, we express it as follows

$$
b_{k}=\sum_{j=1}^{n} a_{k j} x_{j}=\sum_{j=1}^{k-1} a_{k j} x_{j}+\sum_{j=k}^{n} a_{k j} x_{j}
$$

Substituting eqn (24) into the first term of the right-hand side of the above equation yields:

$$
\sum_{j=k}^{n} a_{k j}^{\prime} x_{j}=b_{k}^{\prime}
$$

where

$$
b_{k}^{\prime}=b_{k}-\sum_{l=1}^{k-1} a_{k l} b_{l}^{(k-1)}, \quad a_{k j}^{\prime}=a_{k j}-\sum_{l=1}^{k-1} a_{k l} a_{l j}^{(k-1)}
$$

Separating the $k$-th unknown from eqn (26) gives

$$
x_{k}=b_{k}^{(k)}-\sum_{j=k+1}^{n} a_{k j}^{(k)} x_{j}
$$

where

$$
b_{k}^{(k)}=\frac{b_{k}^{\prime}}{a_{k k}^{\prime}}, \quad a_{k j}^{(k)}=\frac{a_{k j}^{\prime}}{a_{k k}^{\prime}} \quad(j=k+1, k+2, \ldots, n)
$$

Then, substituting eqn (28) back to eqn (24), it follows that

$$
x_{i}=b_{i}^{(k)}-\sum_{j=k+1}^{n} a_{i j}^{(k)} x_{j} \quad(i=1,2, \ldots, k-1)
$$


where

$$
\begin{gathered}
b_{i}^{(k)}=b_{i}^{(k-1)}-a_{i k}^{(k-1)} b_{k}^{(k)}, \quad a_{i j}^{(k)}=a_{i j}^{(k-1)}-a_{i k}^{(k-1)} a_{k j}^{(k)} \\
(i=1,2, \ldots, k-1 ; \quad j=k+1, k+2, \cdots, n)
\end{gathered}
$$

Equations (30) and (28) are the new expressions after the treatment of the $k$-th equation, in which the number of unknowns on the right-hand side is reduced by one compared to the expression of the row $k-1$ as shown in eqn (24). When the treatment of the last row of the equation (23) is finished, the unknowns of each right-hand side disappear and the remaining terms become the solutions of the system of equations.

From the derivation procedure of above formulations it can be seen that the features of REBSM can be classified as follows:

(1) Elimination and back-substitution are performed in the same row of the system of equations, easy for use in engineering numerical methods such as in MDBEM.

(2) Data storage requirement for intermediate process is different in different row treatments. For a system with full-populated matrix $A$, the maximum storage occurs in the middle part of $A$ and the required storage size is a quarter of $A$, being the half of what Gaussian elimination method requires.

(3) From eqn (31) it can be seen that only non-zero elements need to be stored for sparse systems, and no symmetrical and definite properties on $A$ are required. Therefore, REBSM is suitable for use in MDBEM.

(4) If the coefficient matrix $A$ is not dominated by the diagonal elements, the value of $a_{k k}^{\prime}$ in eqn (29) may be zero or very small. In this case, pivoting is necessary to ensure an accurate result. This is easy to fulfill. What only need to do is that the maximum element among $a_{k j}^{\prime}(j=k+1, k+2, \ldots, n)$ determined by eqn (27) is picked up and all related elements in this column are exchanged with those in the $k$-th column.

\section{Numerical example}

Based on the method presented in this paper, a code named BERIM has been written and a corrugated sandwich structure subjected to distributed load (Fig.1) has been analyzed. The upper and lower cover plates of the structure are made of nickel alloy with the Poison's ratio $v=0.25$ and Yong's modulus E=50GMPa; the corrugated brackets are made of titanium with material properties of $v=0.25$ and $\mathrm{E}=250 \mathrm{GMPa}$. The length, width and thickness of the plates are $4 \mathrm{~m}, 2 \mathrm{~m}$ and $0.05 \mathrm{~m}$, respectively, and two plates are spaced by $1 \mathrm{~m}$; the thickness and span of brackets are $0.04 \mathrm{~m}$ and $0.8 \mathrm{~m}$, respectively. In computation, the lower cover is fixed and upper cover is subjected to a distributed pressure load of $0.5 \mathrm{MPa}$. The structure is divided into 22 sub-domains, as shown as Fig.1. The surfaces of the structure is discretized into 7808 eight-noded quadratic boundary elements (Fig.2) with 21236 boundary nodes, among which 1782 are common nodes with 


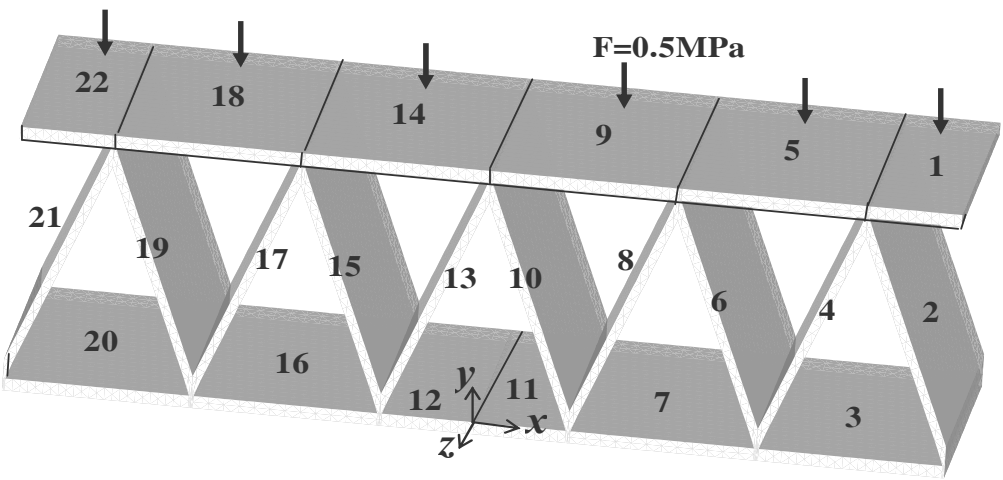

Figure 1: Corrugated sandwich structure under distributed loading.

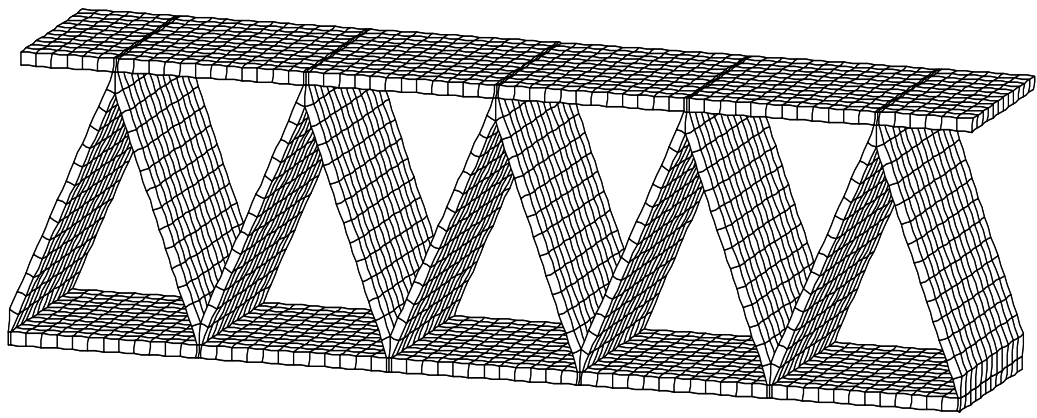

Figure 2: $\quad$ BEM mesh of the corrugated sandwich structure.

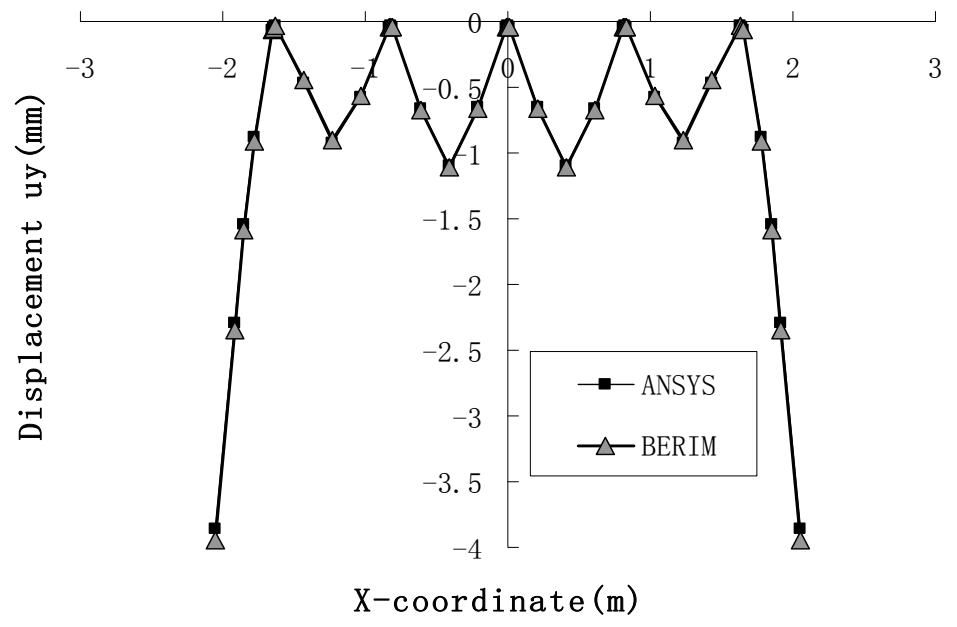

Figure 3: $\quad$ Vertical displacement along mid-line of upper plate surface. 


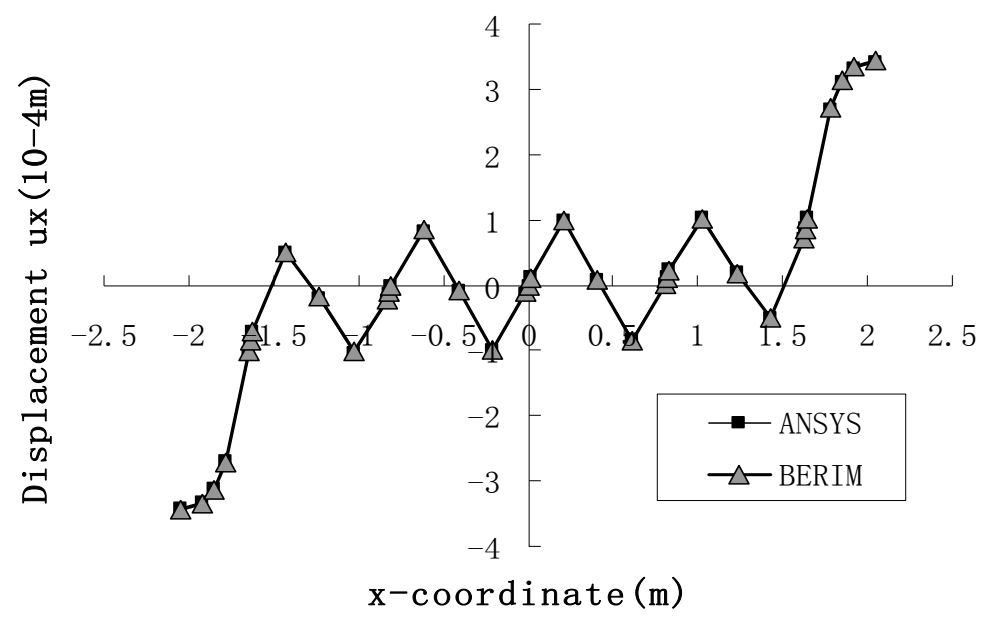

Figure 4: Horizontal displacement along mid-line of upper plate surface.

the degrees of freedom being 5346. The maximum band width of the system of equations is 1743 which shows a higher sparsity. The computational time for solving such system is $107 \mathrm{~s}$ on a PC computer with the CPU of $3.0 \mathrm{GHz}$. For comparison, the problem is also computed using the finite element software ANSYS with the model consisting of 3360 solid 186 brick elements and 3360 nodes. Figs. 3 and 4 show the curves of computed vertical and horizontal displacements along $\mathrm{x}$-direction over the middle line of the upper surface. It can be seen that the current results (BERIM) are in good agreement with those from ANSYS.

\section{Conclusions}

A multi-domain boundary element method has been presented for solving largescale engineering problems. The system of equations assembled using the threestep variable condensing technique has the features of the smallest order and higher sparsity; the row elimination-back-substitution method (REBSM) is an efficient technique for solving non-symmetric and indefinite sparse system of equations, requiring less computer storage and suitable for using in MDBEM.

\section{Acknowledgement}

The authors gratefully acknowledge the National Natural Science Foundation of China for financial support to this work under Grant NSFC No. 10872050.

\section{References}

[1] Brebbia, C.A. \& Dominguez, J. Boundary Elements: an Introductory Course, McGraw-Hill Book Co., London, 1992. 
[2] Luo J. F., Liu Y.J., Berger E. Analysis of two-dimensional thin-structures (from micro- to nano-scales) using the boundary element method. Computational Mechanics, 22, pp.404-412,1998.

[3] J. Wang and X.W. Gao, Structural multi-scale boundary element method based on element subdivision technique, Chinese Journal of Computational Mechanics, 27(2),pp.258-263, 2010.

[4] Kane J.H., Kashava Kumar B.L., Saigal S. An arbitrary condensing, noncondensing solution strategy for large scale, multi-zone boundary element analysis. Comput Meth Appl Mech Eng,79, pp.219-44, 1990.

[5] X.W. Gao, L. Guo, Ch. Zhang, Three-step multi-domain BEM solver for nonhomogeneous material problems, Engineering Analysis with Boundary Elements, 31, pp.965-973,2007.

[6] Ch. Zhang, M. Cui, J. Wang, X.W. Gao, J. Sladek, V. Sladek. 3D crack analysis in functionally graded materials. Engineering Fracture Mechanics, 78,pp.585-604,2011.

[7] X.W. Gao, K. Yang. Thermal stress analysis of functionally graded material structures using boundary element method, Chinese Journal of Theoretical and Applied Mechanics,43(1), pp.136-143, 2011.

[8] Strang, Gilbert. Introduction to Linear Algebra (3rd ed.). Wellesley, Massachusetts: Wellesley-Cambridge Press, pp.74-76, 2003.

[9] Saad Y. Iterative Methods for Sparse Linear Systems. SIAM, Second edition, 2003.

[10] X.W. Gao. Source point isolation boundary element method for solving general anisotropic potential and elastic problems with varying material properties, Engineering Analysis with Boundary Elements, 34, pp.10491057,2010 .

[11] X.W. Gao and T.G. Davies, Boundary Element Programming in Mechanics, Cambridge University Press, 2002.

[12] X.W. Gao. A boundary element method without internal cells for twodimensional and three-dimensional elastoplastic problems. ASME Journal of Applied Mechanics, 69, pp.154-160, 2002. 